\title{
The SSDF Chess Engine Rating List, 2019-12
}

\author{
Lars Sandin and Guy Haworth* \\ Chairman, Svenska schackdatorföreningen; Reading, England
}

\begin{tabular}{|c|c|c|c|c|c|c|}
\hline \# Name & Rating & 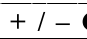 & Games & Win \% & Against & Notes \\
\hline 02 Stockfish 9 x64 $1800 \times 3.6 \mathrm{GHz}$ & 3486 & $28 / 26$ & 802 & $71 \%$ & 3327 & $20-20 v \# 03,21^{1 / 2}-18^{1 / 2} / 2 \# 05,23^{1 / 2}-16^{1} / 2 v \# 06,21^{1} / 2-18^{1 / 2} v \# 08$ \\
\hline 03 Komodo 13.1 x64 1800X $3.6 \mathrm{GHz}$ & 3470 & $32 / 30$ & 520 & $64 \%$ & 3374 & NE; A, Mark Lefler and GM Larry Kaufman; 18-22 v \#01 \\
\hline 04 Komodo 13.02 x64 1800X $3.6 \mathrm{GHz}$ & 3465 & $30 / 29$ & 600 & $65 \%$ & 3354 & $17^{1} / 2-22^{1 / 2} \mathrm{v} \# 01,20^{1 / 2}-19^{1 / 2} \mathrm{v} \# 05,22^{1 / 2-1}-17^{1 / 2} \mathrm{v} \# 06$ \\
\hline 06 Stockfish 9 x64 Q6600 $2.4 \mathrm{GHz}$ & 3449 & $32 / 31$ & 480 & $56 \%$ & 3401 & 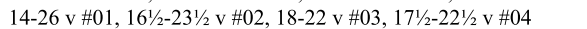 \\
\hline 07 Komodo 12.3 x64 Q6600 $2.4 \mathrm{GHz}$ & 3441 & $38 / 36$ & 360 & $60 \%$ & 3364 & $15^{1 / 2}-24 \frac{1}{2} \mathrm{v} \# 01,17^{1} / 2-22^{1} \frac{1}{2} \mathrm{v} \# 03,17^{1} \frac{1}{2}-22^{1} \frac{1}{2} \mathrm{v} \# 04,20-20 \mathrm{v} \# 06$ \\
\hline 08 Stockfish 8 x64 1800X $3.6 \mathrm{GHz}$ & 3431 & $23 / 21$ & 1260 & $75 \%$ & 3241 & $18 \frac{1}{2}-21 \frac{1}{2} \mathrm{v} \# 02,20-20 \mathrm{v} \# 03,19-21 \mathrm{v} \# 04,17^{1} / 2-22^{1} / 2 \mathrm{v} \# 05$ \\
\hline 09 Stockfish 8 x64 Q6600 $2.4 \mathrm{GHz}$ & 3412 & $31 / 30$ & 560 & $65 \%$ & 3302 & $13^{1} / 2-261 / 2 v \# 01,13 \frac{1}{1} 2-261 / 2 v \# 02,161 / 2-231 \frac{1}{2} \mathrm{v} \# 03,18-22 \mathrm{v} \# 04$ \\
\hline 12 Deep Shredder 13 x64 1800 X $3.6 \mathrm{GHz}$ & 3358 & $24 / 24$ & 880 & $64 \%$ & 3256 & A, Stefan Meyer-Kahlen; $13-27$ v \#02, 14-26 v \#04, 12-28 v \#05 \\
\hline 13 Booot 6.3 .1 x64 1800X $3.6 \mathrm{GHz}$ & 3354 & $24 / 23$ & 843 & $51 \%$ & 3345 & A, Alex Morozov; $10-30 \mathrm{v} \# 01,12-28 \mathrm{v} \# 02,11 \frac{1}{1} 2-281 / 2 \mathrm{v} \# 03$ \\
\hline 14 Komodo 11.01 x64 Q6600 $2.4 \mathrm{GHz}$ & 3343 & $27 / 27$ & 642 & $50 \%$ & 3339 & $9^{1 / 2}-30^{1} \frac{1}{2} \mathrm{v} \# 01,11^{1} \frac{1}{2-2} 28^{1} / 2 \mathrm{v} \# 02,12^{1 / 2}-27^{1} / 2 \mathrm{v} \# 03,16-24 \mathrm{v} \# 04$ \\
\hline 15 Komodo 9.1 x64 Q6600 $2.4 \mathrm{GHz}$ & 3338 & $20 / 19$ & 1475 & $72 \%$ & 3177 & 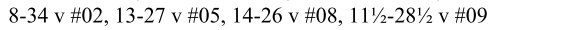 \\
\hline 16 Stockfish 6 x64 Q6600 $2.4 \mathrm{GHz}$ & 3326 & $21 / 20$ & 1256 & $69 \%$ & 3191 & $7-33 \mathrm{v} \# 02,14 \frac{1}{2}-25^{1} / 2 \mathrm{v} \# 08,11^{1 / 2}-28^{1} / 2 \mathrm{v} \# 09,15^{1 / 2}-241 / 2 \mathrm{v} \# 11$ \\
\hline 17 Vajolet2 $2.8 \times 641800 \times 3.6 \mathrm{GHz}$ & 3301 & $61 / 65$ & 123 & $43 \%$ & 3351 & NE; A, Marco Belli; 7-33 v \#01, 12-28 v\#08, 1-2 v\#13 \\
\hline 18 Booot 6.3.1 x64 Q6600 $2.4 \mathrm{GHz}$ & 3299 & $30 / 30$ & 520 & $53 \%$ & 3271 & $8-32 \mathrm{v} \# 01,11-29 \mathrm{v} \# 03,11-29 \mathrm{v} \# 04,9-31 \mathrm{v} \# 05$ \\
\hline 24 Wasp $3.5 \times 641800 \times 3.6 \mathrm{GHz}$ & 3230 & $31 / 33$ & 520 & $32 \%$ & 3359 & A, John Stanback; 5-35 v \#01, 5-35 v \#02, 41/2-351/2 v \#03 \\
\hline 25 Deep Hiarcs 14 1800X $3.6 \mathrm{GHz}$ & 3217 & $26 / 26$ & 720 & $40 \%$ & 3290 & A, Mark Uniacke; $7-33 \mathrm{v} \# 02,7^{1} / 2-32^{1 / 2} / 2 \# 03,5^{1 / 2-34} \frac{1}{2} \mathrm{v} \# 05$ \\
\hline 26 Wasp 3 x64 1800X $3.6 \mathrm{GHz}$ & 3214 & $24 / 25$ & 842 & $39 \%$ & 3292 & 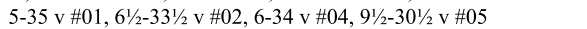 \\
\hline 27 Stockfish 3 x64 Q6600 $2.4 \mathrm{GHz}$ & 3202 & $19 / 18$ & 1420 & $61 \%$ & 3127 & $4 \frac{1}{2}-35^{1} / 2 \mathrm{v} \# 08,15^{1 / 2}-34^{1} / 2 \mathrm{v} \# 11,61 / 2-33^{1} / 2 \mathrm{v} \# 12$ \\
\hline 28 Deep Rybka 4 x64 Q6600 2.4 GHz & 3199 & $20 / 19$ & 1368 & $65 \%$ & 3093 & A, IM Vasil Rajlich; 51/2-341/2 v \#07, 91/2-301/2 v \#11, 8-32 v \#15 \\
\hline 29 Deep Rybka 3 x64 Q6600 2.4 GHz & 3194 & $22 / 21$ & 1371 & $75 \%$ & 3003 & $15^{1 / 2-241 / 2} \mathrm{v} \# 22,21-19 \mathrm{v} \# 2718-22 \mathrm{v} \# 28,22^{1 / 2}-19^{1} / 2 \mathrm{v} \# 30$ \\
\hline 30 Deep Hiarcs 14 Q6600 $2.4 \mathrm{GHz}$ & 3188 & $19 / 18$ & 1450 & $61 \%$ & 3112 & 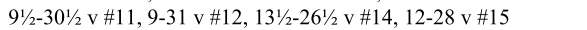 \\
\hline 31 Chiron 3.01 x64 Q6600 $2.4 \mathrm{GHz}$ & 3178 & $27 / 27$ & 656 & $45 \%$ & 3215 & A, Ubaldo Andrea Farina; 7-33 v \#08, 7-33 v \#11 \\
\hline 32 Wasp 3.5 x64 Q6600 $2.4 \mathrm{GHz}$ & 3176 & $41 / 42$ & 280 & $45 \%$ & 3205 & 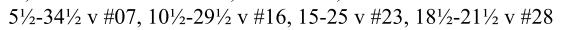 \\
\hline 33 Naum 4.2 x64 Q6600 $2.4 \mathrm{GHz}$ & 3146 & $21 / 21$ & 1123 & $60 \%$ & 3078 & A, Alexander Naumov; $13^{1 / 2-261 / 2} \mathrm{v} \# 19,7^{1} / 2-341 / 2 \mathrm{v} \# 21$ \\
\hline 34 Deep Junior Yokohama x64 Q6600 $2.4 \mathrm{GHz}$ & 3126 & $22 / 22$ & 1010 & $42 \%$ & 3184 & A, Amir Ban \& Shay Bushinsky; 61/2-731/2 v \#08, 6-34 v \#09 \\
\hline 35 Hiarcs 14 Athlon $1.2 \mathrm{GHz}$ & 3100 & $29 / 29$ & 560 & $55 \%$ & 3065 & 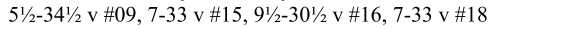 \\
\hline 36 Deep Fritz 13 Q6600 $2.4 \mathrm{GHz}$ & 3097 & $24 / 24$ & 826 & $55 \%$ & 3064 & A, Frans Morsch; 9-31 v \#21, 131/2-261/2 v \#22, 151/2-241/2 v \#27 \\
\hline 37 The Baron 3.43 x64 1800X $3.6 \mathrm{GHz}$ & 3091 & $29 / 31$ & 680 & $26 \%$ & 3272 & A, Richard Pijl; 21/2-371/2 v \#02, 31⁄2-361/2 v \#05, 3-37 v \#08 \\
\hline 48 Millennium ChessGenius Pro M4 120 MHz & 2166 & $59 / 54$ & 160 & $63 \%$ & 2070 & A, Richard Lang; $51 \frac{1}{2}-14^{1} / 2 \vee$ Revelation Ruffian \\
\hline 49 Millennium ChessGenius ARM M4 $48 \mathrm{MHz}$ & 2076 & $51 / 47$ & 211 & $63 \%$ & 1986 & A, Richard Lang; 7-13 v MCG Pro, 8-12 v Montreux \\
\hline 50 Mephisto London $6800012 \mathrm{MHz}$ & 2008 & $59 / 58$ & 140 & $53 \%$ & 1983 & A, Richard Lang; $61 / 2-13 \frac{1}{2}$ v MCG Pro, $10-10$ v MCG \\
\hline
\end{tabular}

Fig. 1. The recently tested 'Selected 50' from SSDF rating list '2019-12' of 2019-12-10, q.v., https://ssdf.bosjo.net. ', 2,3

\footnotetext{
*Corresponding author. E-mail: g.haworth@ reading.ac.uk.

1 'Games' $\equiv$ the number of games, played at ' $40 \mathrm{~m} / 2 \mathrm{hr}+20 \mathrm{~m} / 1 \mathrm{hr}$ ', on which the rating is based. 'Against' $\equiv$ average rating of opponents. ' + ' and '-' denote upper/lower $95 \%$-confidence intervals. 'A' $\equiv$ author(s), 'NE' $\equiv$ new entrant.

${ }^{2}$ Latest platform: AMD Ryzen 7 1800X, 8-core @ 3.6 GHz, 16 GB RAM, SSD, 6-man Syzygy EGTs.

${ }^{3}$ Fuller SSDF data including the '50' and long lists' match detail is available at http://centaur.reading.ac.uk/87834/.
} 